\title{
Kommentare
}

\section{Die Wende im Demonstrationsrecht}

Mit dem vom Bundesjustiz- und Bundesinnenministerium im Juli 1983 vorgelegten (»Kompromiß«-)Vorschlag' für eine Änderung des $\$ 125$ StGB stehen die bislang fehlgeschlagenen Versuche der CDU/CSU in den Jahren $1974,1977^{2}$ und $1981^{3}$, eine Änderung der derzeitigen straf- und versammlungsgesetzlichen Ausgestaltung des Demonstrationsrechts zu erreichen, kurz vor der Vollendung. Ziel ist die Rücknahme wesentlicher Bestandteile der mit dem Dritten Strafrechtsreformgesetz vom 20. 5. 1970 gelungenen Anpassung dieses Rechtsbereichs an die grundgesetzliche Konzeption des Versammlungsrechts. ${ }^{4}$

War Gegenstand insbesondere der Bundesratsinitiative vom Oktober 198 I's ausdrücklich die Änderung auch des Versammlungsgesetzes durch Einfügung eines strafbewehrten Verbots der sogenannten "passiven Bewaffnung " sowie der "Vermummung «, so beschränken sich die Pläne gegenwärtig formell auf eine Änderung des Tatbestandes des Landfriedensbruchs, während die Einführung einer entsprechenden versammlungsgesetzlichen Regelung zur Zeit "geprüft " ${ }^{6}$ wird. In erster Lesung beriet der Bundestag bereits am 17. 9. 1982 einen Gesetzesentwurf des Bundesrates, ${ }^{7}$ mit dem das Antragserfordernis für die Verfolgung von Taten der Sachbeschädigung "aufgelockert« werden soll.

\section{1. Änderung des Straftatbestandes des Landfriedensbruchs (\$ 125 StGB)}

Erster (und symbolträchtigster) Gegenstand der Änderungsbestrebungen ist der Strafbestand des Landfriedensbruchs in seiner seit dem Dritten Strafrechtsreformgesetz vom 20. 5. 1970 geltenden Fassung.

Diese Reform hatte zum Ziel, durch Änderung der einschlägigen strafrechtlichen Bestimmungen den durch das Grundgesetz garantierten demokratischen Rechten der Versammlungs- und Meinungsfreiheit (Art. 8 und , GG) Rechnung zu tragen sowie das StGB auch in diesem Bereich dem rechtsstaatlichen Gebot der Rechtssicherheit adäquat zu gestalten. Insbesondere wurde der potentielle Täterkreis betreffend Landfriedensbruch dadurch eingeschränkt, daß die Begehung von Gewalttätigkeiten nur noch bei nachgewiesener Täterschaft oder Teilnahme tatbestandlich sein sollte. Der Straftatbestand des Auflaufs ( $\$$ I I6 StGB I87I), der die Anwesenheit in einer friedlichen "Ansammlung « für den Fall pönalisierte, daß der behördlichen

\footnotetext{
I Vgl. Frankfurter Rundschau vom 14. 7. 1983, Tagesspiegel vom 8. 7. 1983, Berliner Volksblatt vom 14.7. 1983 , taz vom 8. 7. 1983 , Die Zeit vom 15.7 .1983 .

2 BT-DrS 7/28 54 vom 28. I1. 1974; BT-DrS 7/2772 vom 11. I1. I974; BT-DrS 8/322 vom 26. 4. 1977.

3 BR-DrS $255 / 81$ vor 9. 10. 1981 ; s. auch BT-DrS $9 / 1258$.

4 Vgl. Das Parlament Jg. 1970, Nr. 2, S. I ff.; Nr. 3, S. I ff.; Nr. 8 S. 5 ff.; Nr. 13, S. $9 \mathrm{ff}$

5 S. o. (Fußnote 3).

6 Der Spiegel Nr. 18/1983. Vgl. hierzu auch Frankfurter Rundschau vom 14. 7. 1983 sowie taz vom 14. 7. und 8.7 .1983 .
} 
Aufforderung, sich zu entfernen, nicht Folge geleistet wurde, erfuhr im Zuge der

Reform eine Herabstufung zur Ordnungswidrigkeit ( $\$ \mathrm{I}_{13} \mathrm{OWiG}$ ).

Der nunmehr zwischen Justiz- und Innenministerium ausgehandelte Formulierungsvorschlag für die Neufassung des $\oint_{\text {I }} 25$ Abs. 2 StGB lautet demgegenüber:

„(2) Werden Gewalttätigkeiten (gegen Menschen oder Sachen, d. Verf.) oder Bedrohungen (von Menschen mit einer Gewalttätigkeit, d. Verf.) begangen und fordert ein Träger von Hoheitsbefugnissen

I. die Menschenmenge oder

2. einen bestimmten räumlich abgrenzbaren Teil der Menschen, aus dem diese Handlungen begangen werden, auf, auseinanderzugehen, so wird derjenige, der sich nicht aus der aufgeforderten Menschenmenge entfernt oder sich den der Aufforderung Zuwiderhandelnden anschließt, mit Freiheitsstrafe bis zu einem Jahr oder mit Geldstrafe bestraft.

(3) In den Fällen des Absatzes 2 wird nicht bestraft, wer,

I. ausschließlich dienstliche oder berufliche Pflichten ausübt oder

2. auf die Menschenmenge oder einzelne Personen erweislich einwirkt, um sie davon abzuhalten, Gewalttätigkeiten oder Bedrohungen im Sinne des Absatzes i zu begehen (s. Absatz 2, d. Verf.).

(4) Ist in den Fällen des Absatzes 2 die Schuld des Täters gering, so kann das Gericht von Strafe absehen." ${ }^{8}$

Damit ist das ausschließlich polizeitaktische Problem der Begrenzung des Einsatzes auf »Teilmengen « innerhalb von Großdemonstrationen im Sinne des allein Machbaren und derzeit längst Praktizierten gelöst. Soweit noch im Mai 1983 Divergenzen zwischen Justiz- und Innenminsterium in der Frage bestanden, ob im Falle von sogenannten "Abwieglern « auch formell eine Beweislastumkehr dahingehend stattfinden solle, daß diese sich $»$ nachweislich ${ }^{9}$ mit einer entsprechenden Zielsetzung betätigt haben müssen, ${ }^{10}$ soll ein derartiges Verhalten nunmehr »erweislich «, die Ausübung dienstlicher oder beruflicher Pflichten "ausschließliches "Motiv sein ${ }^{11}$ soweit der "Kompromiß«.

a. Unabhängig von der Möglichkeit einer Begrenzung auf Teilgruppen, ist die tatbestandliche Definition geeignet, mit dem Begriff der "Menschenmenge solche Zusammenkünfte zu erfassen, bei denen es sich um Versammlungen im Sinne des Art. 8 GG handelt, so daß deren Teilnehmer mit Bestrafung zu rechnen haben werden. ${ }^{1 \text { ta }}$

Eine etwaige Unfriedlichkeit der gesamten Demonstration als immanente Grundrechtsschranke des Art. 8 GG kann zumindest nicht schon daraus geschlossen werden, daß es seitens einzelner Personen zu Gewalttätigkeiten kommt. ${ }^{12}$ Hinzutreten muß vielmehr eine Solidarisierung auch der übrigen mit denjenigen, die sich bereits an den Ausschreitungen beteiligen.

Offenkundiger noch, wenn auch in der Sache nicht weitergehend als der im Bundesrats-Entwurf vom Oktober 198 I vorgesehene Tatbestand, ${ }^{13}$ greift die vorliegende

7 BT-DrS 9/1937.

8 Vgl. Frankfurter Rundschau vom 8. 7. 1983 , S. 2.

9 Vgl. taz vom 30.6.1983 und Süddeutsche Zeitung vom 10. 5.1983

10 Vgl. hierzu Tagesspiegel vom 20.5.1983; Frankfurter Rundschau vom 8.6. 1983; taz vom 30. 6. 1983 .

I I S. auch Frankfurter Rundschau vom 14.7. 1983.

i a Vgl. auch Schmude zum Bundesrats-Entwurf vom Oktober 1981, BT, 9. Wahlperiode, 91. Sitzung vom 11. 3. 1982, S. 5467 .

12 Vgl. Herzog, in: Maunz/Dürig/Herzog/Scholz, Grundgesetz-Kommentar Stand November 1982; Art. 8 Rn. 52 sowie Rn. 59; v. Münch, Grundgesetz-Kommentar, 2. Auflage 1981, Art. 8.

13 Während $\$ 125$ Abs. 2 StGB nach dem Bundesrats-Entwurf vom Oktober 198 I (s. o., Fußnote 3) noch voraussetzte, daß die "Menschenmenge» die Gewalttätigkeiten »unterstützen* müsse, steht diese Formulierung gegenwärtig nicht mehr zur Diskussion. In der Sache freilich hat sich hierdurch eine Änderung nicht ergeben, da der Begriff der Unterstützung eine rein subjektive Willensrichtung (so auch Schmude, BR Stenographischer Bericht, 504. Sitzung vom 9. 10. 1981, S. 346) meint, deren Vorhandensein aus der bloßen Existenz der "Menge* geschlossen wird. 
Fassung in den Kernbereich des Art. 8 GG ein, indem eine Willensübereinstimmung mit denjenigen, die Gewalttätigkeiten begehen, aufgrund der bloßen Anwesenheit innerhalb der »Menge « vermutet wird. Diese aber genießst, soweit auch die übrigen Merkmale einer Versammlung vorliegen, es sich daher nicht um eine bloße Ansammlung handelt, den Schutz des Art. 8 GG. Insoweit behält aber auch ein "SichAnschließen « an die (bzw. ein Verbleiben in der) Versammlung strafrechtlich neutralen Charakter.

Die Garantie der Demonstrationsfreiheit wird mit dem neuen $\$ I 25 StGB dadurch unterlaufen, daß Demonstrationen einem polizeilichen Auflösungsvorbehalt unterstellt werden, indem die Bestimmung der immanenten Grenzen der Grundrechtsausübung vollständig in das polizeiliche Ermessen gestellt wird. Der Polizei vor Ort ist damit die Kompetenz eingeräumt, nicht mehr nur, wie bisher, über Verdachtsmomente zu entscheiden und gegebenenfalls nach Aufösung der Demonstration ( $\$$ is VersG) zum Zweck der Strafverfolgung gegen die »Menge« einzuschreiten; vielmehr setzt sie in Zukunft selbst eine Strafbarkeitsbedingung und wird damit in der Lage sein, Versammlungen mit Hilfe einer entsprechenden Aufforderung den Schutz des Art. 8 GG von vornherein zu versagen.

Hinzu kommt, daß insbesondere im Hinblick auf das Tatbestandsmerkmal der Bedrohung von Menschen mit einer Gewalttätigkeit nicht einmal auszuschließen ist, $\mathrm{daß}$ für derartige Aufforderung auch solche Verhaltensweisen zum Anlaß genommen werden, die tatsächlich nicht tatbestandsmäßig sind. Zwar genügt hinsichtlich des Erfordernisses der »zureichenden tatsächlichen Anhaltspunkte «'4 als Voraussetzung für ein Einschreiten der Polizei zum Zweck der Strafverfolgung gemäß $\$$ I 63 StPO formell nicht »eine kriminalistische Hypothese oder eine auf allgemeiner Erfahrung beruhende Verdächtigung, die nicht durch Umstände des konkreten Falles zu belegen ist ". ${ }^{15}$ In Anbetracht der auch für die Polizei als Institution reaktiver strafrechtlicher Sozialkontrolle charakteristischen - da für die Legitimierung und Effektivität der eigenen Tätigkeit erforderlichen - Bildung von Stereotypen, ${ }^{16}$ die die Wahrnehmung realer Geschehnisse entscheidend vorstrukturiert, ist jedoch nicht auszuschließen, daß insoweit ein Bild von Demonstranten als potentielle Gewalttäter für die Verdachtsbildung (mit-)entscheidend ist. ${ }^{17}$ Bestehen aber schon allgemein

14 Kleinknecht, StPO Kommentar, 36. Auflage, 1983, $\$ 163$ Rn. 9.

Is Meyer-Goßner, in: Löwe-Rosenberg, StPO Kommentar, 23. Auflage, 1978, $\$ 163$ Rn. 8 , $\$ 12^{2}$ Rn. 19.

16 Vgl. Fisenberg, Kriminologie, 1979, S. 349 ff., 355.

I 7 Allgemein mag für die Strafverfolgungstätigkeit der Polizei konstitutiv sein, daß sie - im ersten Zugriff objektiv feststellbaren Tatsachen subjektive Momente unterstellt. Es bestehen Anhaltspunkte dafür, daß für die Verdachtsbildung nicht selten äußere Merkmale der verdächrigen Personen entscheidend sind, die unabhängig von einer möglichen Tarbegehung vorliegen, wie etwa das Tragen »schmutziger $\propto$ oder »abgerissener* Kleidung oder als verdächtig perzipiertes Benehmen anläßlich der Konfrontierung mit Polizeikräften (vgl. Feest/Blankenburg, Die Definitionsmacht der Polizei, 1972, S. 35 ff., 37-39). Auf dieser Grundlage wird Realität jeweils entsprechend der Ausgestaltung von Straftatbeständen konstruiert.

Betreffend die spezielle Einsatzsituation anläßlich von Demonstrationen dürfte die Besonderheit eine Rolle spielen, daß dieser kollektiven Form der Meinungsäußerung von vornherein oder erst aufgrund von Einsatzerfahrungen ein ihr angeblich generell immanentes Gefahrenmoment zugeschrieben wird, das dazu dient, eine hohe Intensität präventiver und repressiver Polizeitätigkeit zu rechtfertigen, die zuweilen den legalen Rahmen sprengt. Man denke nur an die allseits bekannten Taschenkontrollen, bei denen "Tatsache« im Sinne des $\$ 23$ Abs. I bln. ASOG oftmals lediglich die beabsichrigte Teilnahme an einer Demonstration ist (vgl. hierzu auch Feest/Blankenburg, a. a. O., S. $4_{4}$ ). Die Problematik der Bildung herabsetzender Stereotypen ist hier noch dadurch verschärft, daß in der polizeilichen Ausbildung Hintergrundinformationen über politisch-soziale Konflikte weitgehend ausgespart werden. Jedenfalls bestehen Anhaltspunkte dafür, daß die - notfalls gewaltsame - Durchsetzung von Sicherheit und Ordnung allein Inhalt und Ziel der Ausbildung darstellt (vgl. Gössner/Herzog, Der Apparat, Ermittlungen in Sachen Polizei, 1982 , S. 78 ff., 8 f f.). Als eine Folge dieser polizeimäßigen Blickverkürzung kann die die Menschenwürde verletzende Bezeichnung des - am 22.9. 1981 im Verlauf des Polizeieinsatzes gegen Demonstrationen aus Anlaß der Räumung besetzter Häuser in Berlin zu Tode gekommenen - Klaus- 
Anhaltspunkte dafür, daß die Polizei Demonstrationen nicht zusätzlich als strafrechtlich neutrale Phänomene wahrzunehmen vermag, so kommt hier noch hinzu, daß bestimmte Protestformen, die sich einerseits nicht in einem nur verbalen Raum halten, andererseits aber auch nicht in Gewalttätigkeiten einmünden, ${ }^{58}$ geeignet sein können, eine bereits bestehende Vermutung, daß eine Bedrohung von Menschen mit einer Gewalttätigkeit vorliege, noch zu verstärken.

Damit wird in zahlreichen Fällen die Annahme einer Bedrohung im Sinne des $\$ 125$ StGB geradezu zwangsläufig sein und eine an keinerlei gesetzliche Voraussetzungen gebundene polizeiliche Auflösung von Demonstrationen zur Folge haben.

Eine derartige Regelung wird gerade Jugendliche, die ihren Protest meist mit größerer Emotionalität zum Ausdruck bringen, in erhöhtem Maße benachteiligen, ${ }^{19}$ indem sie deren Beteiligung am außerparlamentarischen Willensbildungsprozeß in besonderer Weise dem Risiko der Strafverfolgung aussetzt.

b. Zudem bestehen erhebliche Unstimmigkeiten im Verhältnis der geplanten strafrechtlichen Regelung zu den geltenden versammlungsgesetzlichen Vorschriften.

So wird freilich mit der Formulierung: Aufforderung durch einen »Träger von Hoheitsbefugnissen «, auseinanderzugehen, eine Tatbestandsstruktur wieder in das StGB einbezogen, die der des 1970 in veränderter Fassung als $\$ I $_{3}$ OWiG übernommenen $\$ I I 6 StGB I 87 I entspricht. ${ }^{20}$ Dennoch darf die Behauptung mit Skepsis betrachtet werden, derzufolge »der Vorschlag so wirklich Neuartiges überhaupt nicht will ${ }^{21}$

Auffällig ist bereits, daß nach $\mathbb{I}_{\text {I } 3}$ OWiG eine dreimalige Aufforderung erforderlich ist, während in dem vorgeschlagenen Straftatbestand eine einmalige ausreichen soll.

Darüber hinaus läßt der Wortlaut im Unklaren, in welchem Verhältnis überhaupt die Aufforderung, »auseinanderzugehen «, zu den Regelungen des Versammlungsgesetzes steht. Denklogisch ergeben sich nur die Alternativen eines Zusammenhangs entweder mit einem vorausgegangenen Verbot gemäß $₫$ Is Abs. I VersG oder einer Auflösung gemäß Is Abs. 2 VersG. ${ }^{22}$

Jürgen Rattay im Leichenbegleitschein als »Berufsmäßiger Chaot« gelten (vgl. Gössner/Herzog, a. a. O., S. 117 ).

Hinzu kommt schließlich, daß an Demonstrationen im allgemeinen ein Teil der Bevölkerung teilzunehmen pflegt, der sich schon im äußeren Erscheinungsbild von anderen Personen in einer Weise unterscheidet, die sich mit verbreiteten Vorstellungen von Ästhetik und, daraus abgeleitet, einem bestimmten Ordnungsbegriff mitunter nicht verträgt, so daß einem sich selbst nicht retlektierenden (Klein-)Bürgerbewußtsein schnell das Gefühl von Bedrohtheit entstehen mag. Dies könnte ein etwa bei der Polizei vorhandenes Gruppenbewußtsein im Sinne einer "Solidarität gegenüber einer potentiell gefährlichen Außenwelt « (Feest/Blankenburg, a. a. O., S. 26) noch verstärken, das dadurch in noch höherem Maße zur Triebfeder kurzschlüssiger Zuschreibungen geraten kann.

Von Interesse ist in diesem Zusammenhang, daß in der Vergangenheit zuweilen von offiziellen Stellen (Öffentlichkeits-) Vorarbeit dergestalt geleistet worden ist, daß bereits vor geplanten Demonstrationen potentiellen Teilnehmern kriminelle Motive oder generell sozial abweichendes Verhaiten unterstellt worden sind (Gössner/Herzog, a. a. O., S. 89 ff.). Nicht unwesentlich ist auch, inwieweit zutreffende oder schlicht lancierte Informationen (vgl. Gössner/Herzog, a. a. O., S. 92 f.) über geplante Gewalttätigkeiten schon im Vorfeld geeignet sind, Furcht und in der Folge Aggressionen zu verursachen, die den Einsatzstil maßgeblich beeinflussen.

$18 \mathrm{Vgl}$. Grymer, Protest in der jungen Generation als gesellschaftliches Problem, Strafverteidiger 82, $318 \mathrm{ff.}$, $324 \mathrm{f}$.

19 Eisenberg, Minderjährige in der Gesellschaft, 1980, S. 49 f.

$20 \mathrm{Vgl}$. Simson, Verfassungskonforme Demonstrantenbestrafung, ZRP 68 , 10, der - bezogen auf $\$ 116 \mathrm{StGB}$ 1871 - das Versammlungsgesetz grundsätzlich auch noch nach Auflösung der Versammlung für die Spezialregelung hält.

2. So aber Scholz zum Bundesrats-Entwurf vom Oktober ${ }_{198}$ r, BR Stenographischer Bericht, $50_{4}$. Sitzung vom 9. 10. 198 I, S. 344 .

22 Bereits die systematische Einordnung der Vorschrift ist danach ungewöhnlich. Während bislang die Ausgestaltung der Rechtsfolgen bei Teilnahme an einer verbotenen bzw. Verbleiben in einer aufgelösten Versammlung gemäß $\$ 29$ Abs. I Nr. I, 2 VersG der gesetzlichen Wertung entsprechen mag, daß hier in 
Mit dieser Formulierung aus dem OWiG enthält der Vorschlag einen - im Hinblick sowohl auf den Bestimmtheitsgrundsatz (Art. 103 Abs. 2 GG) als auch die Regelung des $\mathbb{\int}$ IS Vers $G$ - problematischen Präszisionsmangel. So wird nicht einmal dem engen strafrechtlichen Rechtmäßigkeitsbegriff, der vor allem an die korrekte Beurteilung der Zuständigkeit für bestimmte Diensthandlungen anknüpft, Rechnung getragen, wie denn auch überhaupt nach dem Wortlaut des geplanten $\ 125$ Abs. 2 StGB das Erfordernis der Rechtmäßigkeit der Aufforderung entfällt - eine Begrenzung, die dem Gesetzgeber 1970 bei zahlreichen Tatbeständen (vgl. z. B. $\$$ I I 3 Abs. 3, 4 StGB) unter rechtsstaatlichen Aspekten unerläßlich schien. ${ }^{23}$

Danach kann sich die Merkwürdigkeit ergeben, daß eine Ordnungswidrigkeit nach $\$ 29$ Abs. I Nr. 2 VersG i. V. m. $\$$ Is Abs. 2 VersG mangels Zuständigkeit der die Auflösung verfügenden Behörde nicht vorliegt, wohl aber der Tatbestand des $\$ I 25 StGB erfüllt ist. Ist die Auflösungsverfügung gar wegen Evidenz der Unzuständigkeit im Sinne des $\$ 44$ Abs. I WvVfG nichtig und damit unwirksam $(\$ 43$ Abs. 3 $\left.V_{w} V f G\right)$, so muß ( $\left.\$ 16_{3} S t P O\right)$ dennoch bei entsprechender Aufforderung gegen die Versammlung eingeschritten werden, was faktisch der Durchsetzung der nichtigen Auflösungsverfügung gleichkommt. Schließlich hat die Polizei nach der bisherigen Rechtslage selbst noch in den Fällen, in denen eine unmittelbare Gefährdung der öffentlichen Sicherheit tatsächlich gegeben und die Auflösungsverfügung materiell rechtmäßig ist, Entschließungsermessen (vgl. $\$ 29$ Abs. 1 Nr. 2 VersG i. V.m. $\$ 53$ $O W_{i G}$ ). Nach der neuen Regelung fordert das Legalitätsprinzip ( $\$ 16_{3}$ Abs. I StPO) unbedingt auch dann ein polizeiliches Einschreiten, wenn materiell lediglich eine Putativgefahr oder zumindest nicht eine unmittelbare Gefährdung der öffentlichen Sicherheit vorliegt. ${ }^{24}$

c. Zum Zweck der erleichterten Feststellung der "Tatbeiträge der Hauptakteure ${ }^{25}$ schon diese Formulierung ist geeignet, den übrigen die Rolle freiwilliger Statisten zuzuschreiben - soll künftig von dem Erfordernis des Nachweises einer Tatbeteiligung abgesehen werden.

Eine derartige Mißachtung der Unschuldsvermutung (Art. 6 Abs. 2 MRK) ${ }^{26}$ steht allerdings nicht stets im Widerspruch zu der Rechtsprechung. Mit dem extensiven Täterbegriff sowie der strafrechtsdogmatischen Kategorie der psychischen Beihilfe hat diese eine Begrifflichkeit geschaffen, die es ihr gestattet, je nach »Judiz « im Einzelfall vom Vorliegen volitiver Momente überzeugt ( $\$ 26$ I StPO) zu sein, die hier allein die Strafbarkeit begründen. ${ }^{27}$

Bestraft werden sollen nach dem neuen $\$ 125$ StGB zwar nicht solche Personen, die mausschließlich in Ausübung dienstlicher oder beruflicher Pflichten handeln, sowie

der Hauptsache nicht Störer im polizeirechtlichen Sinne, sondern Notstandspflichtige betroffen sind, die schon nach allgemeinem Polizeirecht eine Privilegierung genießen $\left(\$_{13}\right.$ bln. ASOG), verkehrt die geplante Regelung diese Wertung in ihr Gegenteil. Auch unter diesem Aspekt begegnet die Auffassung, derzufolge der Gesetzgeber nicht gehindert sei, Nichtstörer im Sinne des Polizeirechts "als Störer « zu betrachten (vgl. Herzog, a. a. O., (Fußnote I2), Art. 8 Rn. 91) dem Einwand einer Verletzung des Übermaßverbots.

23 Vgl. etwa Diemer-Nicolaus, in: Das Parlament, Jg. 1970, Nr. 8, S. 7.

24 Letztere Möglichkeit ist bereits im Tatbestand des $\$ 125$ StGB angelegt, der mit der Formulierung "... Gewalttätigkeiten ... oder Bedrohungen ... in einer die öfentliche Sicherbeit gefäbrdenden Weise * (Hervorhebung d. Verf.) einen Pleonasmus insoweit enthält, als diese Verhaltensweisen mit einer Gefährdung von Rechtsgütern einzelner auch die öffentliche Sicherheit gefährden. Der Tatbestand mag damit materiell ein minus gegenüber $₫$ is VersG darstellen.

$25 \mathrm{Vgi.} \mathrm{BR-DrS} 255_{1} 8_{1}$, Anlage, S. 8.

26 Vgl. auch Frankenberg, »Landfriede* und Demonstrationsfreiheit, KJ 8 I, 269 ff., zu den Vorgängen um die Nürnberger Massenverhaftung vom 5. 3. 1981 ; s. auch Die Zeit vom 15.7. 1983.

27 Zur (tatbestandsorientierten) Reduktion von Realität vgl.: Eisenberg, a. a. O. (Fußnote I6), S. 146; zu den verfahrensrechtlichen Auswirkungen dieser Rechtsprechung im Sinne einer ermittlungsbehördlichen Antizipation einer späteren Verurteilung vgl. Frankenberg, a, a. O. (Fußnote 26), S. 271-274. 
nicht diejenigen, die als sogenannte "Abwiegler « »erweislich « auf andere einwirken, »um sie davon abzuhalten, Gewalttätigkeiten oder Bedrohungen von Menschen mit einer Gewalttätigkeit zu begehen«. Da aber zum einen der Begriff der »Unterstützung « eben nicht Handlungen bezeichnet, die - wie etwa das»Anheizen « anderer zur Begehung von Gewalttätigkeiten - schon bislang dem Tatbestand unterfallen, sondern lediglich eine nicht objektivierte, rein subjektive Willensrichtung ${ }^{28}$ meint, besteht die Gefahr, daß Pressevertreter, die der polizeilichen Aufforderung nicht Folge leisten nicht mehr "ausschließlich" in Ausübung "berufliche(r) « Pflichten handeln und dann mit Bestrafung zu rechnen haben werden; hinzu kommt, daß demnach künftig die Polizei vor Ort in eigener Machtvollkommenheit im Rahmen ihrer Strafverfolgungstätigkeit darüber wird entscheiden können, ob sie die Presse als wohl wesentlichsten Faktor öffentlicher Kontrolle anläßlich von Polizeieinsätzen gegen Demonstranten zuzulassen bereit ist. ${ }^{29}$ Sogenannte "Abwiegler « werden schließlich außerordentlich weitgehende Aktivitäten entfalten müssen, um straflos auszugehen: $\mathrm{Da}$ nämlich einerseits eine Erweiterung des Tatbestandes angestrebt ist, andererseits aber nach der bisherigen Rechtsprechung für die Annahme etwa einer psychischen Beihilfe - unter Ausblendung der Kausalitätsfrage ${ }^{30}$ - bereits die bloße Anwesenheit bei der Tatbegehung ${ }^{31}$ selbst dann genügen soll, wenn sich der Betreffende zugunsten des Opfers einsetzt, ${ }^{32}$ dürften künftig für eine Straflosigkeit noch weit darüber hinausgehende Handlungen erforderlich sein, etwa solche, die das Vorgehen der Polizei gegenüber Demonstranten unterstützen. ${ }^{32 a}$ Insofern wird es auch kaum einen Unterschied machen, ob es tatbestandlich erforderlich ist, daß die Handlungen von "Abwieglern«»erweislich« stattgefunden haben. Art. 8 GG erhält dadurch folgende (ungeschriebene) Fassung:

"Alle Deutschen haben das Recht, sich ohne Anmeldung oder Erlaubnis friedlich, ohne Waffen und vor allem so zu versammein, daß nicht der Eindruck entsteht, sie würden ihre allgemeine Pflicht zur Verbrechensbekämpfung vernachlässigen.*

Vorliegende Ausnahmeregelung konkretisiert demnach zugleich den Begriff der Friedlichkeit im Sinne des Art. 8 GG dergestalt, daß denjenigen von vornherein der Grundrechtsschutz versagt ist, deren Verhalten lediglich fiktiven Handlungspflichten nicht entspricht. Denkbar ist derartiges überhaupt nur auf der Grundlage der Lehre von einer - aus den Art. 18, 21 Abs. 2, 9 Abs. 2 GG hergeleiteten - allgemeinen Mißbrauchsschranke der Grundrechte. ${ }^{33}$ Indem hier unter Umkehrung des Verhältnisses von Grundrechtsschutz als Regel- und Grundrechtsverwirkung als Ausnahmefall134 ursprünglich legale Grundrechtsausübung in den strafrechtlich rele-

28 Schmude, a. a. O. (Fußnote ${ }_{13}$ ).

29 Vgl. hierzu Gössner/Herzog, a. a. O. (Fußnote 17), S. so ff., $322 \mathrm{ff}$.

30 Kritisch: Rudolphi/Horn/Samson/Schreiber, Systematischer Kommentar, Bd. I, 2. Auflage 1977, \27 Rn. 14 .

31 Vgl. auch BGH NJW 72, 1366; 72, 1571; 75, 49 f.; 78, 816; a. A. neuerdings AG Freiburg NStZ 82, 247.

32 BGH bei Dallinger MDR 67, 173; vgl. auch Frankenberg, a. a. O. (Fußnote 26), S. 278

32a Zur Statuierung einer derartigen Hilfspflicht kritisch: Frankenberg, a. a. O. (Fußnote 26), S. 279 unter Hinweis auf die $\int S 138,258$, i45 d. StGB sowie Schnoor, BR Stenographischer Bericht, $\mathrm{SO}_{4}$ Sitzung vom 9. 10. 1981, S. 34 I: "Garantenpflicht « für fremdes (!) gefährdendes Vorverhalten. Vgl. auch den beim Polizeipräsidenten in Berlin verfaßten Entwurf einer Neufassung des $\$ 125$ StGB vom 14. 1. 1982 (Dir VB c-O $7702 / 1$, S. 2), in dem die mangelnde Bereitschaft »zur aktiven Mitwirkung bei der Abwehr, zur Zusammenarbeit mit der Polizei« beklagt wird.

33 Vgl. BVerfG NJW 75, 1641 sowie Merten, Demonstration unter dem Gesetz, Aus Politik und Zeitgeschichte, 10/69, S. 21 ff., 25 f.; kritisch: v. Greiff, Staatsphilosophie - eine bisher unbekannte Form der Innenpolitik (Anmerkung zu OVG Berlin KJ 77, 76 ff.), in: KJ 77, 8 r ff.; Denninger, Freiheitsordnung Wertordnung - Pflichtordnung, JZ 75, $545 \mathrm{ff}$.

34 Kritisch hierzu: Preuß, Legalität und Pluralismus, 1. Auflage 1973 , S. 117 ff., I $23 \mathrm{ff}$; Ridder, Die soziale Ordnung des Grundgesetzes, 1975 , S. $54 \mathrm{ff}$., $134 \mathrm{ff}$. 
nerell Auswirkungen auf sicherheits- und ordnungspolitische Belange habe, die allein eine Einschränkung des Grundrechts aus Art. 8 GG rechtfertigen würden, ${ }^{43} \mathrm{im}$ Hinblick auf die Wahrheitsermittlungspflicht (auch) des Gesetzgebers ${ }^{44}$ erhebliche Bedenken.

Erscheint dem Entwurf das Mitführen der genannten Gegenstände bereits als »typische Vorbereitungshandlung "45 zur Beteiligung an gewaltsamen Ausschreitungen, so steht dem entgegen, daß es gerade friedlichen Demonstranten unter den obwaltenden Verhältnissen als ein Gebot der Vernunft erscheinen muß, Vorkehrungen zu treffen, die zumindest einen minimalen Schutz der Körperintegrität - sei es vor der polizeilichen Anwendung unmittelbaren Zwangs im Falle der Begehung von Gewalttätigkeiten seitens anderer Versammlungsteilnehmer, sei es vor Übergriffen etwaiger Gegendemonstranten - gewährleisten. ${ }^{46}$ Diesem Verhalten, dem eine Aggressivtendenz nicht innewohnt, wird daher schon der Begriff der "passiven Bewaffnung " nicht gerecht.

Inwieweit das Erfordernis des subjektiven Bestimmungszwecks ein Korrektiv gegenüber einer polizeilichen Inanspruchnahme solcher Personen zu bilden vermag, die gewaltsame Auseinandersetzung nicht anstreben, ist überaus zweifelhaft. Der vom Polizeipräsidenten in Berlin verfaßte Entwurf zur Verschärfung des Demonstrationsrechts vom 14. Januar 1982,47 nach dem die Pönalisierung des Mitführens von Selbstschutzvorrichtungen in den Tatbestand des Landfriedensbruchs selbst übernommen werden sollte, mag als Indiz gelten, daß mit derartigen Gegenständen ausgerüstete Personen von polizeilicher Seite grundsätzlich kriminelle Motive unterstellt werden. "Passiv bewaffnete« Personen gelten danach nicht als Demonstranten, sondern als "potentielle Gewalttäter«, deren »Aktionen ... überhaupt keine Beziehung zum Versammlungsrecht $\star^{4}{ }^{8}$ hätten. Das Mitführen von Selbstschutzvorrich-

43 Insoweit erfordert verfassungskonformes Verständnis der Versammlungsfreiheit als politisches Teilhaberecht im Sinne des Demokratieprinzips (Art. 20 Abs. I, 2 Satz 1 GG) eine Qualifizierung des - nach dem Wortlaut des Art. 8 Abs. 2 GG - einfachen Gesetzesvorbehalts (vgl. Ott, Gesetz über Versammlungen und Aufzüge Kommentar, 3. Auflage 1979, S. 26 Rn. 7; ders.: Das Recht auf freie Demonstration, 2. Auflage 1969, S. $28 \mathrm{ff}$., $38 \mathrm{ff}$.). Eine gegenteilige Auffassung (Merten, a. a. O. (Fußnote 33)), nach der die Versammlungsfreiheit - vergleichbar der freien Berufsausübung (Art. 12 Abs. 1 Satz 2 GG) - unter Beachtung allgemeiner Verfassungsprinzipien schon aufgrund "vernünftiger Erwägungen des Gemeinwohls « einschränkbar sei, kann nicht überzeugen. Schon die Berufung auf die Bannmeilengesetze des Bundes und der Länder (Merten, a. a. O., S. 29) geht fehl, da es sich bei den Gesetzgebungsorganen um Einrichtungen des Staates handelt, die Schutzgut der öffentlichen Sicherheit sind, und es sich insofern bei den Bannmeilengesetzen um Konkretisierungen des Gesetzesvorbehalts handelt, die inhaltlich über nvernünftige Erwägungen des Gemeinwohls « hinausgreifen (vgl. Götz, Allgemeines Polizei- und Ordnungsrecht, 7. Auflage 1982, S. 39 f.). Auch die Entstehungsgeschichte ist hier nicht eindeutig, wie sich aus der erst in der vierten Lesung des Grundgesetz-Entwurfs vom Hauptausschuß des Parlamentarischen Rates ohne Begründung vorgenommenen Streichung des Art. 8 Abs. 2 Satz 2 ergibt, gemäß dem ein Verbot von Versammlungen nur »bei unmittelbarer Gefahr für die öffentliche Sicherheit « ergehen können sollte (vgl. Parlamentarischer Rat, Verhandlungen des Hauptausschusses, Bonn 1948/49, S. 746; Parlamentarischer Rat, Grundgesetz für die Bundesrepublik Deutschland (Entwürfe), Bonn 1948/49, S. 198, 242). Schließlich würde sich eine solche Interpretation - wenngleich sanktionslos - dem Vorwurf des Völkerrechtsverstoßes aussetzen. Art. 2 I des Internationalen Pakts über bürgerliche und politische Rechte enthält die - seit 1976 auch für die Bundesrepublik verbindliche - Regelung, daß nur solche Einschränkungen der Versammlungsfreiheit zulässig sind, "die in einer demokratischen Gesellschaft im Interesse der nationalen oder der öffentlichen Sicherheit, der öffentlichen Sittlichkeit und zum Schutz der Rechte und Freiheiten anderer notwendig sind «.

44 Noll, Gesetzgebungslehre, 1973, S. 86 ff., 94 f.; zur Problematik des (formellen) Unterschiedes der Wahrheitsermittlungspflicht bei Legislative und Judikative, vgl. Eisenberg, a. a. O. (Fußnote 16), S. 142 m. w. N. sowie S. 146

45 BR-DrS 25s/81, Anlage, S. 5

46 Anschaulich: Gössner/Zerzog, a. a. O. (Fußnote 17), S. 108-1 I 2; vgl. auch Ott, Demonstrationsrecht im Zwielicht, DuR 82, $186 \mathrm{ff}$. sowie Bergerowski, BT 9. Wahlperiode, 91. Sitzung vom I1.3. 1982, S. 5464 .

47 S. o. (Fußnote 32a)

48 S. o. (Fußnote 32a), S. 2 (Hervorhebung d. Verf.). 
tungen sei »eindeutig erkennbare Vorbereitungshandlung « ${ }^{49}$ für die Begehung von

Straftaten seitens derjenigen, die "offensichtlich die Wahrnehmung der aus der Versammlungsfreiheit fließenden Rechte mißbrauchen « wollten. ${ }^{50}$

Allgemein spricht eine Vermutung dafür, daß polizeiliche Einsatzerfahrungen das Blickfeld insoweit verengen, als sich gerade diejenigen Demonstrationen besonders einprägen, in deren Verlauf es zu gewaltsamen Auseinandersetzungen kommt und bei denen tatsächlich der überwiegende Teil der schließlich Festgenommenen derartige Gegenstände mitführt. Die Tatsache, daß ein gleiches auch bei friedlich verlaufenden Demonstrationen der Fall ist, ist der Wahrnehmung weitgehend entzogen, da es hier zu einer Konfrontation ja nicht kommt.

So betrachtet liegt die Annahme nicht fern, daß bei Neufassung des $₫$ I2S StGB ein entsprechendes Erscheinungsbild schon für die Verdachtsbildung im Rahmen dieser Bestimmung entscheidend sein wird und solchermaßen ausgerüstete Personen bevorzugt Strafverfolgungsmaßnahmen ausgesetzt sein werden, so daß es eines selbständigen Verbots der sogenannten "Passivbewaffnung" gar nicht mehr bedarf.

Schließlich sind auch abgesehen von diesbezüglich zu erwartenden Zuschreibungen jedenfalls die Befugnisse zu beachten, die der Polizei gemäß $\ 163$ a StPO selbst gegenüber unverdächtigen Personen zustehen.

Die Normierung eines abstrakten Gefährdungstatbestandes räumt der polizeilichen Definitionsmacht einen derart weiten Spielraum ein, daß Demonstranten, von denen eine Gefährdung der öffentlichen Sicherheit im Sinne einer konkreten Bedrohung von Individualrechtsgütern's nicht ausgeht, zu Störern bzw. Straftätern umdefiniert werden können. Mit dieser Konsequenz befindet sich der Entwurf in Einklang mit der - in den 7oer Jahren mit dem polizeilichen Präventionskonzept der "Inneren Sicherheit « ${ }^{{ }^{2}}$ eingeleiteten - Entwicklung, polizeiliche Eingriffsbefugnisse mittels abstrakter Gefährdungstatbestände in das Vorfeld des normrelevanten Bereichs zu verlagern, ${ }^{33}$ und verdeutlicht damit exemplarisch die immense Gefahr der Aushöh-

49 S. o. (Fußnote 32a), S. 2 (Hervorhebung d. Verf.).

so S. o. (Fußnote 32a), S. I (Hervorhebung d. Verf.)

Vgl. neuerdings auch Scholz, Rechtsfrieden im Rechtsstaat, NJW 83, 705 ff., (709), der einerseits zutreffend auf die Bedeutung der Grundrechte für den Minderheitenschutz hinweist, andererseits Grundrechtsmißbrauch durch Minderheiten »sogar durch Gewalttätigkeiten * (Hervorhebung d. Verf.) befürchtet.

51 Der vorliegende Entwurf zeigt einmal mehr, daß das Demonstrationsrecht als "Element des status constituens« (vgl. Frankenberg, Demonstrationsfreiheit - eine verfassungsrechtliche Skizze, KJ 8 r, 370 ff., (372); Denninger, Demonstrationsfreiheit und Polizeigewalt, in: Polizei und Strafprozeß im demokratischen Rechtsstaat, Hrsg. Denninger/Lüderssen, 1978, S. 127 ff., 133) eine verfassungskonforme Einschränkung des Schutzguts der öffentlichen Sicherheit, das allgemein gleichgesetzt wird mit der objektiven Rechtsordnung, dahingehend verlangt, daß lediglich der von der Verfassung selbst gebotene Schutz konkreter Individualrechtsgüter kollektiven, die demokratische Willensbildung (mit-)konstituierenden politischen Aktionen Schranken zu setzen vermag (so Frankenberg, a. a. O., unter Hinweis auf Art. 79 Abs. 3 i. V. m. Art. 20 Abs. 2 Satz I GG sowie den demokratischen Grundsatz des Minderheitsschutzes). Deutlich wird dies insbesondere, wenn man die geplanten Regelungen im Zusammenhang mit den versammlungsgesetzlichen Verbots- und Auflösungsbefugnissen betrachtet: Folgt man nämlich auch für den Bereich des Demonstrationsrechts der herkömmlichen Auffassung, derzufolge ein Verstoß gegen ein gesetzliches Verbot immer auch zugleich eine Störung der öffentlichen Sicherheit darstellt, so wird mit der Normierung eines abstrakten Gefährdungstatbestandes nicht zuletzt die Regelung des $\$$ is Vers $G$ unterlaufen, die gerade gewährleiten soll, daß polizeiliche Eingriffe nur äußerstenfalls in Betracht kommen (vgl. auch allgemein Wagner, Polizeirecht, 1982, S. 59: "Doppelfunktion der Strafnormen «).

§2 Vgl. hierzu Wagner, a. a. O. (Fußnote 51), S. 24 ff. sowie Gössner/Herzog, a. a. O. (Fußnote 17), S. $314 \mathrm{ff}$., die auf die Funktion dieses Präventionsmodells hinweisen, die insbesondere darin besteht, Auswirkungen von Legitimitätsschwächen des politischen Systems in der Folge ökonomischer Krisen vorzubeugen; s. auch Blumenwitz, Versammlungsfreiheit und polizeiliche Gefahrenabwehr bei Demonstrationen, in: Festschrift für Samper, 1982, S. I 31 ff., sowie Schreiber, Duldung von Rechtsbrüchen, ebenda, S. I 7 ff., die übereinstimmend einen mangelnden semotionalen(!) Bezug des Bürgers zu Recht und Staat « befürchten.

53 S. auch Riehle, Sicherheit im Vorfeld des Rechts, KrimJ 82, I67 ff., I 73 f. sowie Gössner/Herzog, a. a. O. (Fußnote 17 ), S. 316 ff., unter Hinweis auf die Problematik der nachträglichen Legalisierung zunächst 
lung von Grundrechtsgarantien, die in der Abkehr von dem überkommenen polizeirechtlichen Begriff der konkreten Gefahr liegt. Im Bereich des Demonstrationsrechts wird Grundrechtsschutz künftig abhängen von individueller Risikobereitschaft.

Eine Regelung, die potentielle Demonstranten auf die Alternative verweist, entweder unter Gefährdung der Körperintegrität an Versammlungen teilzunehmen ${ }^{54}$ oder auf die Ausübung des Grundrechts aus Art. 8 GG zu verzichten, wird praktisch überwiegend die Nichtwahrnehmung des Demonstrationsrechts aufgrund der begründeten Furcht vor staatlicher Sanktionierung zur Folge haben und tangiert damit Art. 8 GG in seinem Wesensgehalt (Art. 19 Abs. 2 GG)."s

An der Verfassungswidrigkeit der geplanten Regelung kann auch der Ermächtigungsvorbehalt ( $\$ 1$ 7a Nr. I letzter Halbsatz: »Niemand darf . . Gegenstände ... mit sich führen, ohne behördlich ermächtigt zu sein «) nicht vorbeiführen. Materiell handelt es sich hierbei um ein präventives Verbot mit Erlaubnisvorbehalt. Ein solches ist nach dem Wortlaut des Art. 8 Abs. 2 GG zwar zulässig. Indem eine Enumeration der Ermächtigungsgründe jedoch nicht stattfindet, vermag es - in Anbetracht der für die Verwirklichung des Demokratiegebots konstitutiven Bedeutung der Versammlungsfreiheit ${ }^{56}$ - diese in ihrem Kern nicht zu gewährleisten, da damit die Grundrechtsausübung als solche aufgrund des weiten Ermessensspielraums der Behörde in Frage gestellt sein wird.

Als besonders problematisch erweist sich zudem der Erlaubnisvorbehalt bei Spontandemonstrationen, also in den Fällen, in denen die Befürworter einer solchen Regelung einen Vorteil der abstrakt-generellen Regelung gegenüber der bereits nach geltendem Recht möglichen Auflagenerteilung erblicken, ${ }^{37}$ soweit eine behördliche Ermächtigung sich hier nur konkludent aus einem Nicht-Einschreiten der Polizei als zuständiger Behörde ( $\$ 4$ Abs. I bln. ASOG) ergeben kann. Da das Vorliegen einer Ermächtigung, wie etwa in $₫ 27$ Vers $G^{58}$ sowie in $₫ 53$ Abs. I Nr. I WaffG ${ }^{59}$ als Tatbestandsmerkmal anzusehen ist, wird künftig in einem solchen Fall das Verhalten der Polizei vor Ort über die Strafbarkeit entscheiden.

b. In solchen Fällen dagegen, in denen Demonstranten dem geplanten Verbot ent-

rechtswidriger, aber Effektivitätserfordernissen der Polizei entsprechender Praktiken; über Parallelitäten zur Terroristengesetzgebung: Bergerowski, a. a. O. (Fußnote 46 ), S. 5462 f. sowie Pensky, ebenda, S. 5459 .

54 So auch Ott, a. a. O. (Fußnote 46 ), S. 188

is $\mathrm{D}$ a mithin durch die geplante Regelung jedenfalls der Verhältnismäßigkeitsgrundsatz verletzt wird, kann hier bei der Frage der Grenzen der Wesensgehaltsgarantie dahinstehen, ob Grundrechte einen unantastbaren absoluten Wesensgehalt aufweisen oder der Kern jeweils durch Vergleich mit den kollidierenden öffentlichen Interessen im Rahmen einer Verhältnismäßigkeitsprüfung zu ermitteln ist (vgl. BVerfGE 22, $219 ; 27,351 ; 30,53)$. In der Sache eine Vorwegnahme der geplanten Regelung enthält z. B. die badenwürttembergische "Verwaltungsvorschrift des Innenministeriums zur Durchführung des Versammlungsgesetzes« vom 10.6. 1981, die praktisch für den Regelfall eine entsprechende Auflage empfiehlt (Abschnitt II Nr. 3.4.). Allgemein mag dies die Folge sein, wenn die Rechtsprechung Zweifel an der Rechtmäßigkeit einer Auflagenerteilung gemäß $\$ is Abs. I Vers $G$ mit der Begründung zurückweist, mim Hinblick auf die gerichtsbekannten Vorfälle bei unfriedlich verlaufenden Demonstrationen in letzter Zeit" (so VG Berlin NVwZ 82, 268), sei die Erwartung eines gewaltsamen Verlaufs gerechtfertigt, und damit die Beweisanforderungen betreffend eine unmittelbare Gefährdung der öffentlichen Sicherheit auf Null reduziert. In diesem Zusammenhang ist die Annahme von Bedeutung, daß die im Prozeß reaktiver strafrechtlicher Sozialkontrolle den übrigen in der zeitlichen Abfolge vorgeordneten Institutionen die Tendenz haben, behördeninternen Effektivitätskriterien entsprechend (Eisenberg, a. a. O. (Fußnote 16), S. 349 f.), die Praxis der nachgeordneten Behörden zu antizipieren, so daß künftig die Erteilung einer solchen Auflage auch dann zu besorgen sein wird, wenn eine Gefahr im Sinne des $\$ 15$ Abs. I VersG nicht nachweislich vorliegt.

s6 BVerfGE 7, 199.

57 Miltner, BT 9. Wahlperiode 9r. Sitzung vom ir. 3. 1982, S. 5459 sowie Bohl, ebenda, S. 5469 ; s. zuletzt auch Scholz, a. a. O. (Fußnote 50), S. 712.

58 Dietel/Gintzel, Demonstrations- und Versammlungsfreiheit Kommentar, 7. Auflage, 1982, $\$ 27$ Rn. 3. \$9 Potrykus/Steindorf, Waffenrecht Kommentar, s. Auflage 1982, $\$ 53$ Anm. a. 
sprechend Selbstschutzvorrichtungen nicht mitführen, es aber dennoch zu einer

Auflösung der Versammlung gemäß \Is VersG - etwa wegen Verstoßes gegen das Verbot der "Passivbewaffnung " seitens anderer Personen ${ }^{60}-$ kommt, oder z. B. an die Demonstration eine Aufforderung gemäß (dem geplanten) $\$ 125 StGB ergeht, sind sicherheits- und ordnungspolizeiliche Belange im Sinne der polizeilichen Generalklausel nur vermittelt betroffen. Zwar wird ein Polizeieinsatz zum Zweck der Zerstreuung der verbleibenden »Menschenmenge mit den Mitteln unmittelbaren Zwangs dann besonders effektiv sein, wenn Demonstranten Mittel nicht zur Verfügung stehen, um - in Anbetracht der zunehmend gefährlicheren Bewaffnung der Polize $^{6 \mathrm{r}}-\mathrm{zu}$ erwartenden erheblichen Verletzungen zu entgehen, und dadurch mittelbar der (Wieder-)Herstellung des Rechtssicherheitsgefüls derjenigen dienen, die "jede kollektive Aktion, weil es zu Ausschreitungen kommen könnte, als bedrohlich (empfinden) «. ${ }^{62}$

Nicht aber rechtfertigt eine solche Erleichterung polizeilicher Aufgaben eine damit eintretende Tangierung des Wesensgehalts des Art. 2 Abs. 2 Satz ${ }_{I}$ GG $^{6}{ }^{63}$

c. Schließlich begegnet die vorgeschlagene Fassung des $\$ 1$ i a Nr. 1 VersG dem Einwand mangelnder Bestimmtheit (Art. 103 Abs. 2 GG), insofern als dem objektiven Betrachter nahezu jedes Kleidungsstück yom Schutzhelm bis zum Regenmantel als

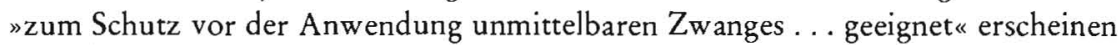
kann. ${ }^{64}$

Jedenfalls wird die Vorschrift - in Anbetracht der zu erwartenden Einlassung, ein derartiger Schutz sei nicht bezweckt gewesen - zumindest bei Beachtung rechtsstaatlicher Grundsätze kaum anwendbar sein. ${ }^{65}$

\section{Strafbewehrtes 》Vermummungsverbot«(\$17a Nr. 2 i. V.m. $\int 27$ Abs. 2 VersG)}

Nach dem im Bundesrats-Entwurf vom 9. I0. 198 I vorgesehenen \ 17a Nr. 2 VersG darf bei Demonstrationen »niemand . . . sich maskieren oder sonst unkenntlich machen, es sei denn, daß die zuständige Behörde dies zugelassen hat, weil ein friedlicher

60 S. o. (Fußnote $5 \mathrm{I}$ ).

6i S. hierzu Gössner/Herzog, a. a. O. (Fußnote 17), S. 290 ff., 3 roff., unter Hinweis auf die völkerrechtliche Ächtung des Einsatzes von Giftgasen im Krieg durch die Genfer Konvention vom 17.6. 1925, die aus Anlaß des Einsatzes dieser Gase in Vietnam von der Vollversammlung der Vereinten Nationen im Jahre 1969 ausdrücklich unter Einbeziehung von Tränengasstoffen bestätigt wurde; generell ablehnend gegenüber einer »völkerrechtlichen Dimension « im Bereich der Innenpolitik: Scholz, a. a. O. (Fußnote 50), S. 706: „Für das Verhältnis Staat und Bürger gilt dagegen anderes «; im Ergebnis ebenso Blumenwitz, a. a. O. (Fußnote 52), der zwar einerseits auf die besondere Gefahr des Einsatzes von CS-Reizstoffen für "Asthma- und Bronchitiskranke (S. ISI) sowie darauf hinweist, daß »in der Praxis... die tödliche Wirkung meist nur von der Konzentration des Wirkstoffs (abhängt) (S. I 49), andererseits aber nweitergehende Beeinträchtigungen « von Demonstranten für in der Regel ausgeschlossen hält. Demgegenüber zeigen gerade jüngere Erfahrungen mit dem Einsatz dieser Giftgase durch Wasserwerfer, daß insbesondere bei niedrigen Außentemperaturen die Konzentration überhaupt nicht kontrollierbar ist, da sich diese Wirkstoffe nicht gleichmäßig mit Wasser verbinden.

62 Frankenberg, a. a. O. (Fußnote SI), S. 382.

63 So auch Ott, a. a. O. (Fußnote 46), S. I 88, vgl. die Resolution des 17. Kongresses der Gewerkschaft der Polizei (GdP) (Frankfurter Rundschau vom 9. 10. 1982), die die Entwicklung sogenannter "polizeitypischer Einsatzmittel" befürwortet, die die „Lücke zwischen Schlagstock und Pistole schließen«, und gleichzeitig ein - freilich nicht strafbewehrtes - Verbot des Mitführens von Selbstschutzvorrichtungen fordert. Vgl. auch Frankfurter Rundschau v. 25. 7. 1983 zur Ankündigung der Einführung sogenannter "Wuchtgeschosse" durch den Bundesinnenminister.

Nicht auszuschließen ist demgegenüber, - man denke an das zum Schutz vor Tränengas mit reizstoffneutralisierenden Stoffen präparierte Halstuch -, daß ein Verbot des Mitführens von Selbstschutzvorrichtungen Versammlungsteilnehmern das Befolgen der ihnen durch die geplante Neufassung des strafrechtlichen Tatbestandes des Landfriedensbruchs auferlegten Rechtspflicht des Sich-Entfernens im Falle gewaltsamer Auseinandersetzungen erheblich erschweren, wenn nicht gar unmöglich machen wird.

64 So auch Schnoor, BR Stenographischer Bericht, 504. Sitzung vom 9. 10. 1981, S. 340.

65 So auch Bergerowski, a. a. O. (Fußnote ${ }_{46} 6$ ), S. $5463 \mathrm{f}$. 
Verlauf der Veranstaltung gewährleistet ist, oder daß es sich um herkömmliche Veranstaltungen, insbesondere Volksfeste handelt, bei denen maskierte Personen teilzunehmen pflegen «.

a. Einmal abgesehen davon, daß eine Abgrenzung gegenüber Selbstschutzvorrichtungen, wie z. B. Motorradhelmen, im allgemeinen nicht zweifelsfrei erfolgen können wird (Art. $\left.{ }_{103} A b s .2 G G\right){ }^{66}$ ist der in der Entwurfsbegründung genannte Begriff der $V$ ermummung ${ }^{{ }^{67} 7}$ auch in anderer Hinsicht sprachlich unpräzise. Ungeachtet ferner sprachästhetischer Gesichtspunkte, über die man streiten könnte, erinnert er weniger an typische Vorbereitungshandlungen zur Beteiligung an gewaltsamen Ausschreitungen, denn an - die Art der Bekleidung betreffende - Vorkehrungen zum Schutz vor Erkältungskrankheiten, die vernünftigerweise bei einem Aufenthalt im Freien bei winterlichen Temperaturen getroffen werden, wenngleich auch hier nicht zu verkennen ist, daß Personen, bei denen eine Bereitschaft zur Begehung von Gewalttätigkeiten vorhanden ist, ein Interesse daran haben mögen, unerkannt zu bleiben.

Aber auch der in $₫ 1$ i a Nr. 2 VersG selbst genannte Begriff der Maskierung weckt zunächst Assoziationen betreffend das traditionell geforderte Erscheinungsbild von Personen anläßlich karnevalistischer Festivitäten, die ja - bürgernah - von dem Verbot der Maskierung gerade nicht erfaßt sein werden.

b. Daß die Pönalisierung lediglich solcher Verhaltensweisen angestrebt sei, die der Verhinderung einer späteren Identifizierung im Rahmen einer Strafverfolgung wegen mutmaßlicher Teilnahme an der Begehung von Gewalttätigkeiten dienen und das Verbot dadurch ausschließlich gewaltsamen Ausschreitungen vorbeugen soll, läßt der geplante $\ 17 \mathrm{a} \mathrm{Nr}$. 2 VersG nicht erkennen.

Nur die ungeschminkte Meinungsäußerung soll danach nicht die öffentliche Sicherheit gefährden. In der Tendenz entspricht dies durchaus dem - dem Versammlungsgesetz zugrundeliegenden - Bild von Demonstrationen als von festgefügten politischen Organisationen straff organisierten Umzügen, denen Kriterium des Erfolges wie auch Medium der Meinungsäußerung vor allem die Zahl der Versammelten ist, ${ }^{68}$ das aber grundsätzlich nicht auf die eher dramatisierenden ${ }^{69}$ Formen der Meinungskundgabe zugeschnitten ist, die sich Ende der 6oer, Anfang der 7oer Jahre herausgebildet haben.

Auf einem bestimmten Arrangement von Maskierung und Kostümierung beruhende Medien kollektiver Meinungskundgabe zu schaffen, mag aber sowohl den Versuch gerade jugendlicher Personen widerspiegeln, die strikte Trennung zwischen Rationalität politischen Engagements und - als unpolitisch oder gar politikfeindlich definierter - Emotionen aufzuheben ${ }^{7 \circ}$ als auch als eine der wenigen Möglichkeiten wahrgenommen werden, einen - bei den Adressaten vermuteten oder real vorhandenen ideologischen "Grauschleier « überhaupt durchdringen zu können. ${ }^{71}$

Indem auch derart phantasievolle Demonstrationsformen dem Verbot subsumierbar sind, werden einer Generation, die - nicht ohne Grund dem institutionalisierten Krisenmanagement mißtrauend - mit großem politischen Verantwortungsbewußt-

66 Ott, a. a. O. (Fußnote 46 ), S. I $88 \mathrm{f}$.

67 BR-DrS 255/8 I Anlage, S. 4.

$68 \mathrm{Vgl}$. Schily/Becker/Geulen, Stellungnahme in dem Verfassungsbeschwerdeverfahren betreffend dasVerbot von Demonstrationen gegen die Errichtung des Kernkraftwerks Brokdorf, I BvR 233/81, I BvR $341 / 8$ I, S. I 2-15 m. w. N

69 Frankenberg, a. a. O. (Fußnote 51), S. 382

$70 \mathrm{Vgl}$. Grymer, a. a. O. (Fußnote 18 ), S. 324 f. Dabei mag es sich um einen Spezialfall des Problems der besonderen Ausgestaltung von Straftatbeständen - aufgrund erhöhrer Normrelevanz und sozialer Sichtbarkeit jugendgemäßer Verhaltensweisen handeln (vgl. Eisenberg, a. a. O. (Fußnote 19), S. 49 f.).

71 Vgl. auch Frankenberg, a. a. O. (Fußnote 51), S. 376. 
sein angetreten ist, die Lösung substantieller Lebensprobleme der Gesellschaft (mit-) zu gestalten, dafür grundlegende Instrumente aus der Hand genommen. Eine solche Regelung, die faktisch bestimmte moderate Formen politischer Auseinandersetzung oktroyierte, könnte außerparlamentarischem Protest schließlich derart die Schärfe nehmen, daß er in Funktion und Zielsetzung insgesamt neutralisiert würde.

Im übrigen ist durchaus nicht unwesentlich, inwieweit für ein Verhalten, das dazu dient, eine Identifizierung aufgrund etwa von Fotografien zu verhindern, das - u. a. durch den Radikalen-Erlaß geschaffene - innenpolitische Klima in der Perzeption der meist jungendlichen Demonstranten entscheidend ist..$^{72}$ Diesen mag es überwiegend keineswegs als eine "falsche Propaganda " erscheinen, "daß staatliche Stellen Demonstranten erfassen und Lichtbilder mit nachteiligen Folgen auswerten ${ }^{73}$

Durch dieses Prisma betrachtet ist die beabsichtigte Unerkennbarkeit nicht notwendig Vorbereitung zu strafbaren Handlungen, sondern schafft in zahlreichen Fällen erst die Voraussetzung für die Grundrechtsausübung..$^{74}$

Da mithin zum einen die Maskierung Instrument der politischen Meinungsäußerung sein kann, zum anderen aber auch unabhängig davon tatsächlich oder vermeintlich mit der Grundrechtsausübung verbundene Risiken vermeiden hilft, führt auch hier

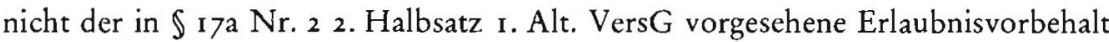
daran vorbei, daß die geplante Regelung Art. 8 GG in seinem Wesensgebalt berührt (Art. 29 Abs. 2 GG).7s

Es mag dahinstehen, ob - wie die Literatur zum Teil empfiehlt - eine abstraktgenerelle Regelung dieser Art zulässig wäre unter der Voraussetzung, daß »objektive Umstände bzw. objektivierbare Erfahrungen mit vergleichbaren Demonstrationen den Rechtsbruch wabrscheinlich machen " ${ }^{76}$ Mit einer Ausnahme ausschließlich für solche Versammlungen, bei denen »ein friedlicher Verlauf ... gewährleistet ist«, kehrt sich jedenfalls die derzeitig geltende Beweislastverteilung vollständig um: Nicht mehr hat die Behörde die Wahrscheinlichkeit eines unfriedlichen Verlaufs

72 Vgl. auch Bergerowski, a. a. O. (Fußnote 46 ), S. 5463.

73 So aber der Polizeipräsident in Berlin, a. a. O. (Fußnote 32a), S. 8.

74 Demnach ist ein Unerkanntbleiben nicht nur Voraussetzung für die Ausübung des Demonstrationsrechts seitens ausländischer Personen (Art. 2 Abs. I GG), insbesondere soweit es sich um Emigranten aus totalitären Staaten handelt (s. hierzu Ott, a. a. O. (Fußnote 46), S. 189). Gerade solche Grundrechte, die wie das Demonstrationsrecht - politische Kommunikation in der Öffentlichkeit gewährleisten, sind durch (potentielle) staatliche Informationseingriffe besonders gefährdet (vgl. Schmidt, Die bedrohte Entscheidungsfreiheit, $\mathrm{JZ} 74,244 \mathrm{ff} ., 247 \mathrm{f}$.). Die vorbeugende Überwachung legaler politischer Betätigungen ist grundsätzlich rechtswidrig, da hierdurch zum einen $m$ Bedingungen geschaffen (werden), die vom Grundrechtsträger wegen des damit verbundenen psychologischen Drucks als nachteilig empfunden werden und daher geeignet sind, ihn von der Grundrechtsausübung abzuhalten " (VG Köln JZ 82, 8 s5 ff., 857 ); zum anderen fehlt es in dem hier interessierenden Zusammenhang für derartige Grundrechtseingriffe (Art. 2 Abs. 1 i. V.m. Art. I Abs. I GG) (vgl. VG Köln a. a. O.) an jeglicher Rechtsgrundlage. Soweit in der Entwurfsbegründung vor allem darauf abgestellt ist, mit dem "Vermummungsverbot Strafverfolgungsmaßnahmen zu erleichtern, ist zu berücksichtigen, daß eine Rechtsgrundlage, die der Polizei eine Observation von Personen zum Zweck der Datenerhebung gestattet, die aufgrund ihres Eingriffscharakters nur aufgrund spezialgesetzlicher Ermächtigung zulässig ist, nicht existiert. Als bloße Aufgabenzuweisungsnorm kann hierfür auch nicht $\$ 16_{3}$ StPO herangezogen werden (a. A.: Ahlf, Der Begriff des »Eingriffs $s_{*}$ insbesondere bei kriminalpolizeilicher Tätigkeit und die sog. »Schwellentheorie zu $\$$ I63 Abs. I StPO, Die Polizei 83, 4 I ff., der unter Bezugnahme auf die Wesentlichkeitstheorie(!) Informationseingriffe für nicht erheblich und daher $\$$ I $_{3} \mathrm{Abs}$. I StPO als Rechtsgrundlage für ausreichend hält.) Diese Auffassung begegnet schon deshalb erheblichen Bedenken, weil die Wesentlichkeitstheorie gerade entwickelt wurde, um dem Gesetzesvorbehalt auch bei exekutiven Grundrechtseinschränkungen in sogenannten »besonderen Gewaltverhältnissen «(!) Geltung zu verschaffen (vgl. z. B. BVerwG NJW 75, 1182; BVerfG NJW 75, ז 879; OVG Münster JZ 76, 273 ff.; VGH Mannheim NJW 76, 869). Darüber hinaus ist betreffend die Tatsache der Datenerhebung ein qualitativer Unterschied der hier interessierenden im Verhältnis zu den in den $\$ \$ 8 \mathrm{Ib}, 100 \mathrm{a}$ StPO geregelten Eingriffen nicht zu erkennen; vielmehr steht hinsichtlich der mangelnden Kontrollmöglichkeit durch den Betroffenen der Informationseingriff - etwa durch Beobachtung - nahe dem in $§ 100$ a StPO geregelten Eingriff.

75 S. o. Fußnote 55 .

76 Herzog, a. a. O. (Fußnote I2), Art. 8 Rn. 61. 
darzutun, sondern potentielle Demonstranten tragen die Beweislast betreffend die Voraussetzungen uneingeschränkter Grundrechtsausübung. Darüber hinaus sind nicht einmal die objektiven Umstände benannt, unter denen von der Friedlichkeit von Versammlungen auszugehen ist. Auch hier entscheidet demnach die Verwaltung nach freiem Ermessen. Außerdem besteht die Gefahr, daß mit einer solchen Formulierung derart hohe Anforderungen an den Nachweis über das Vorliegen der Voraussetzungen einer Erlaubniserteilung gestellt werden, daß eine $»$ Unbedenklichkeitserklärung « seitens der Behörden kaum einmal wird erfolgen können. Denn begegnet unter den derzeitigen Verhältnissen bereits die Prognose eines gewaltsamen Demonstrationsverlaufs merheblichen Unsicherheiten und Beweisschwierigkeiten ",7 so liegt die Vermutung nahe, daß der Vorbehalt des friedlichen Verlaufs in der Praxis leerliefe. ${ }^{78}$

c. Der (Nicht-)Zulassung nach freiem behördlichem Ermessen unterliegen schließlich gerade künstlerische Formen politischer Meinungsäußerung, sogenannte engagierte Kunst - man denke etwa an die Interpretation des Gedichts „Freiheit und Democracy " von Bertolt Brecht in Form des "Anachronistischen Zuges «, die sich neben sonstiger Medien des Straßentheaters auch einer Maskierung der Darsteller bedient -, so daß das Verbot auch Art. s Abs. 3 Satz I GG in seinem Wesensgehalt berübren wird. ${ }^{79}$

d. Festzuhalten bleibt, daß die geplanten - abstrakte Gefährdungstatbestände normierenden - Regelungen geeignet sind, gerade die den demokratischen Willensbildungsprozeß konstituierenden Grundrechte in ihrem Bestand zu gefährden.

Generell bestehen im übrigen Anhaltspunkte dafür, daß es zu den latenten Funktionen des Entwurfs des Bundesrates zahlt, die Praxis zu veranlassen, polizeiliche Befugnisse im Präventivbereich in einer Weise auszuweiten, die zuweilen den legalen Rahmen sprengt. Daß in der Vergangenheit ein Sonderrecht für bestimmte Gruppen potentieller Demonstranten geschaffen worden ist, belegt die außerordentlich großzügige Handhabung des Vorbeugegewahrsams (vgl. \$ I $8 \mathrm{Nr}$. I bln. ASOG) ${ }^{80}$ die in

77 BR-DrS $255 / 8$ I, Anlage, S. 6; vgl. hierzu Gintzel, Vermeintliche bzw. tatsächliche Regelungslücken im Versammlungsrecht und im Strafrecht als Ursache polizeilicher Einsatzprobleme. Die Polizei 82, 299 ff., der darauf hinweist, daß der Anteil der gerichtlich nicht bestätigten versammlungsrechtlichen Auflagen an der Gesamtzahl entsprechender Verfügungen in den Jahren 1979 bis 1981 im Bundesgebiet und in Berlin (West) lediglich i Promille betrug.

78 So auch Ott, a. a. O. (Fußnote 46), S. $188 \mathrm{f}$.

79 Entgegen VGH München NJW 81, 2428 f, der hierin eine künstlerische Darstellung nicht zu sehen vermag, wird man solchen Formen künstlerisch-satirischer Stellungnahme den Schutz des Art. s Abs. Satz I GG nicht versagen dürfen. Die Begründung, Elemente politischer Meinungsäußerung würden derart im Vordergrund stehen, daß die Kunstfreiheitsgarantie nicht betroffen sein könnte, überzeugt schon insoweit nicht, als die Annahme eines Ausschlußverhältnisses zwischen Kunst und politischer Äußerung fehlgeht. Anderenfalls würde der gesamte Bereich kabarettistischer Darbietungen sowie auch yon Karikaturen mit politischem Inhalt als Formen politischer Meinungsäußerungen, die sich des Mittels künstlerischer Darstellung bedient, nicht dem Schutzbereich des Art. S Abs. 3 Satz I GG unterliegen (vgl. Ott, Versammlungsfreiheit contra Kunstfreiheit?, NJW 8r, 2397 f., kritisch auch gegenüber einem objektiven Kunstbegriff; vgl. auch BVerfGE $30,173,188 \mathrm{f}$.).

80 Nach Primärerfahrungen aus der Praxis soll z. B. zuweilen die vorbeugende Ingewahrsamnahme von Personen mit der Annahme einer Wiederholungsgefahr aufgrund eines nicht einmal abgeurteilten früheren Demonstrationsdelikts begründet worden sein. Vgl. auch Frankfurter Rundschau v. 25. 7. 1983 zur Wuppertaler "Ausforschungsrazzia«. Von Interesse ist hier auch die vorbeugende Ingewahrsamnahme zah|reicher Personen vor der Demonstration anläßlich des Besuchs des US-Außenministers in Berlin am 1 3.9. 1981. Einen Zusammenhang zwischen vorausgegangenen Aufrufen zu "unfriedlichen Aktionen " und den am Nollendorfplatz versammelten Personen und damit eine gegenwärtige erhebliche Gefahr im Sinne des $\$$ I $8 \mathrm{Nr}$. I bln. ASOG sieht der Klageabweisungsantrag des Polizeipräsidenten(VG 1 A 26/82) vom 31.3.1982 allein in deren näußerem Erscheinungsbild (Ausrüstung mit Helmen, Vermummung, bemalte Gesichter) sowie (dem) räumlichen Zusammenhaltu (Klageabweisungsantrag, a. a. O., S. 2); vgl, auch die Resolution des 17 . Kongresses der GdP (s. o. Fußnote 63), in der darauf hingewiesen ist, daß ein Verbot von Maskierung und Passivbewaffnung der Polizei Maßnahmen schon im Vorfeld von Demonstrationen ermögliche. 
der Sache nicht weniger bedeutet als die faktische Aberkennung von Grundrechten. ${ }^{81}$ Von Interesse ist in diesem Zusammenhang etwa auch die Beschlußempfehlung des Innenausschusses vom i s. 9. 1982 zum vorliegenden Entwurf, ${ }^{82}$ in der die ablehnende Stellungnahme damit begründet wird, »das yorhandene Instrumentarium (sei) tauglich und wirksam ..., wenn man von ihm besonderen Gebrauch mache . .. ${ }^{83}$ Jener Tendenz entspricht es schließlich, wenn die Gewerkschaft der Polizei die »meldedienstliche Erfassung der Straftäter ${ }^{84}$ befürwortet, um gegen diese sodann "dezentrale kleine und frühzeitige Präventivmaßnahmen ${ }^{85}$ zu ermöglichen.

Es ist dies ein weiterer Anwendungsfall einer - durch die Lehre allgemeiner Mißbrauchsschranken der Grundrechte legitimierten - Tendenz, die sich dadurch auszeichnet, daß Einzelpersonen in dem Maße der Grundrechtsschutz versagt wird, in dem legale, aber funktionswidrige Grundrechtsausübung ein reibungsloses Funktionieren des politischen Prozesses gefährdet. ${ }^{86}$

Sabine Wolski

Bemerkenswert ist außerdem, daß der $\mathrm{K}$ lageabweisungsantrag erwähnt, die $\mathrm{K}$ lägerin sei mbereits am 22. 6. 198 I bei einer Durchsuchung unter den Besetzern des Hauses Eisenacher Str. $\mathrm{O}_{3} 3$ in Schöneberg angetroffen worden « (ebenda, S. 3). Darüber hinaus sei sie »beim Herannahen von Einsatzkräften der Polizei ... mit weiteren weiblichen Personen zum U-Bahneingang an der südlichen Seite des Nollendorfplatzes" (ebenda, S. 4) gelaufen.

81 Verschärft wird diese Problematik dadurch, daß Personen, die von einer auf richterlicher Anordnung beruhenden Ingewahrsamnahme nach $\$ 18 \mathrm{Nr}$. I bln. ASOG betroffen sind, weitergehender Rechtsschutz versagt wird (vg]. LG Berlin 84 T XX B $43 / 82$ vom 22. 3. 1982; BayObLGZ 1971 , 1 83 , I85). Diese Privilegierung der polizeilichen Freiheitsentziehung ohne richterliche Bestätigung gegenüber der richterlich angeordneten trägt der Bedeutung des Grundrechts aus Art. 2 Abs. 2 Satz 2 GG sowie den Art. ${ }_{10}$ Abs. 2, 19 Abs. 4 GG (vgl. auch BVerwG NJW 82, 536f.) nur unzureichend Rechnung. Indem den Betroffenen unter Ablehnung einer Analogie zur verwaltungsgerichtlichen Fortsetzungsfeststellungsklage eine Rehabilitation versagt wird, besteht die Gefahr, daß sich damit die Annahme der Voraussetzungen des $\$$ i $8 \mathrm{Nr}$. I bln. ASOG für vergleichbare Situationen perpetuiert und demzufolge diese Personen auch künftig mit vorbeugender Ingewahrsamnahme zu rechnen haben werden.

82 BT-DrS 9/1985 vom 15.9 . 1982 .

83 Ebenda, S. 4 (Hervorhebung d. Verf.).

84 Demonstrationen und kriminelle Begleiterscheinungen der Gegenwart, Leitsätze und Forderungen der Gewerkschaft der Polizei vom 22. 4. 1981 , S. 7 .

8 s Ebenda, S. 7.

86 S. hierzu Preuß, Die Aufrüstung der Normalität, in: Kursbuch 56 (1979), S. Is ff., 29 ff. Unter dem Gesichtspunkt der zunehmenden Resonanz außerparlamentarischer Protestbewegungen in der Bevölkerung einerseits (eindrucksvoll hierzu: Stümper, Der polizeiliche Grundauftrag - auf dem Hintergrund einer prognostischen Einschätzung künftiger Entwicklungen, Die Polizei 82, I ff.), einer reduzierten inneren Stabilität und Integrationsfähigkeit des politischen Systems der Bundesrepublik und Berlin (West) andererseits ist bemerkenswert die ausdrückliche Ausblendung der Kostenfrage im BundesratsEntwurf vom Oktober 1981 (BT-DrS 255/81, S. 1: ... D. Kosten: Keine); dies zumal, wenn man berücksichtigt, daß der Gesetzgeber noch mit dem Dritten Strafrechtsformgesetz von $1970-$ also nach Beendigung der ersten allgemeinen Überproduktionskrise in der Geschichte der Bundesrepublik und bevor noch die damalige Studentenbewegung an Bedeutung verloren hatte - nicht zuletzt aufgrund von Kosten- und Kapazitätserwägungen das Forum zur Artikulation demokratischen Dissenses erweitert hat. 